\title{
Circulating periostin levels increase in association with bone density loss and healing progression during the early phase of hip fracture in Chinese older women
}

\author{
M. Farrokhi ${ }^{1} \cdot$ D. Arjaki $^{2} \cdot$ F. Peykanpour ${ }^{3}$ \\ Received: 20 December 2017 / Accepted: 2 January 2018 / Published online: 21 August 2020 \\ (C) International Osteoporosis Foundation and National Osteoporosis Foundation 2020
}

Dear Editor,

We read with great interest a recent article by Yan et al. [1] entitled "Circulating periostin levels increase in association with bone density loss and healing progression during the early phase of hip fracture in Chinese older women." In this article, the authors aimed to investigate clinical characteristics, bone mineral density (BMD), and bone turnover markers after fracture within 2 days and also follow up sPostn levels during 1 year after 2 days in patients with osteoporotic hip fracture. As stated in the statistical analysis section of the article, they used covariance (ANCOVA) or Mann-Whitney $U$ test to assess statistical changes in concentrations of sPostn in 1 year. Similar to one-way analysis of variance (ANOVA), the oneway ANCOVA is used to evaluate significant differences in numerical dependent variable between two or more independent (unrelated) groups [2-5]. Furthermore, Mann-Whitney $U$ test is used to determine whether there are any significant differences in numerical dependent variables with nonnormal distribution between two independent (unrelated) groups [2-5]. The authors investigated differences in concentrations of sPostn in one sample of cases in different timepoints using ANCOVA. Furthermore, they used MannWhitney $U$ test for comparison of concentration of sPostn between two time-points of measurement in one sample of the cases. Therefore, the authors must use repeated measures ANCOVA instead of ANCOVA for comparison of sPostn concentrations between different time-points. Moreover, they must use the Wilcoxon test instead of the Mann-Whitney $U$

M. Farrokhi

mehrdadfarokhi72@yahoo.com

1 Dr. Farrokhi Eris Research Institute, Tehran, Iran

2 Shahrekord University of Medical Sciences, Shahrekord, Iran

3 School of Dentistry, Qom University of Medical Sciences, Qom, Iran test for comparison of sPostn concentrations between two time-points of measurement.

We also have another comment from a statistical point of view. The authors investigated the percent changes of sPostn, Beta isomerized C-terminal crosslinking to type I collagen (BCTX), and N-terminal procollagen of type I collage (PINP) during different time-points. However, they did not provide explanatory importance from a statistical point of view. Therefore, in a similar way, they can use the Wilcoxon test and repeated measures ANCOVA or repeated measures ANOVA for comparison of percent changes of sPostn, BCTX, and PINP between different time-points of measurement to evaluate significant differences.

\section{Compliance with ethical standards}

Conflict of interest None.

\section{References}

1. Yan J, Liu H, Li H, Chen L, Bian Y, Zhao B, Han H, Han S, Han L, Wang D (2017) Circulating periostin levels increase in association with bone density loss and healing progression during the early phase of hip fracture in Chinese older women. Osteoporos Int:1-7

2. Gaddis ML (1998) Statistical methodology: IV. Analysis of variance, analysis of co variance, and multivariate analysis of variance. Acad Emerg Med 5(3):258-265

3. Farrokhi M (2017) Sema3A and multiple sclerosis. Gene 615:41

4. Farrokhi M, Shirian N (2017) Statistical comments on "no seasonal variation in physical activity of Han Chinese living in Beijing". Int J Behav Nutr Phys Act 14(1):151

5. Farrokhi M, Arjaki D (2017) Statistical comments on "cytokine and chemokine profiles in patients with neuromyelitis optica spectrum disorder". Neuroimmunomodulation 24(2):120-120

Publisher's note Springer Nature remains neutral with regard to jurisdictional claims in published maps and institutional affiliations. 